\title{
On stability of 3-manifolds
}

by

Sławomir Kwasik (New Orleans, LA) and Witold Rosicki (Gdańsk)

\begin{abstract}
We address the following question: How different can closed, oriented 3-manifolds be if they become homeomorphic after taking a product with a sphere?

For geometric 3-manifolds this paper provides a complete answer to this question. For possibly non-geometric 3-manifolds, we establish results which concern 3-manifolds with finite fundamental group (i.e., 3-dimensional fake spherical space forms) and compare these results with results involving fake spherical space forms of higher dimensions.
\end{abstract}

The purpose of this paper is to study the following stability problem:

Problem. Let $M^{3}, N^{3}$ be closed oriented 3-manifolds. Assume that $M^{3} \times S^{n}$ and $N^{3} \times S^{n}$ are homeomorphic (i.e., $M^{3} \times S^{n} \approx N^{3} \times S^{n}$ ) for some $n \geq 1$. Are $M^{3}$ and $N^{3}$ homeomorphic?

It is not difficult to show that if $\Sigma^{3}$ is a homotopy 3-sphere, then $\Sigma^{3} \times S^{n}$ $\approx S^{3} \times S^{n}$ for any $n \geq 1$. Therefore, to avoid the unresolved status of the Poincaré conjecture, until the final section we assume first that 3-manifolds considered in this paper are geometric in the sense of Thurston (cf. [T]). The well-known conjecture of Thurston asserts that all 3-manifolds are geometric. Also, for simplicity, we assume all 3-manifolds to be prime (cf. [H]). Given that, the results of this paper give a complete solution to the stability problem.

Stability by $S^{1}$. It turns out that in terms of the behavior under stabilization by $S^{1}$ one class of 3 -manifolds stands out. It is a subclass of Seifert fibered 3 -manifolds. This was already noticed by V. Turaev in [Tu], namely

THEOREM 1. Let $M^{3}, N^{3}$ be closed oriented geometric 3-manifolds. Then $M^{3} \times S^{1} \approx N^{3} \times S^{1}$ is equivalent to $M^{3} \approx N^{3}$ unless $M^{3}$ and $N^{3}$ are

2000 Mathematics Subject Classification: Primary 58R80, 57S25.

Research supported by the NSF Grant INT-9811590.

Supported by KBN:127/E-335/5/99 and Gdańsk University Grant BW/5100-S-0201-0. 
Seifert fibered 3-manifolds which are surface bundles over $S^{1}$ with periodic monodromy and surface genus $>1$.

A short sketch of proof of the above theorem was presented in $[\mathrm{Tu}]$. For the convenience of the reader, we insert a detailed proof of Theorem 1, different from Turaev's sketch, in the appendix of this paper. Our proof contains a discussion of the case of "small" Seifert manifolds, which was not discussed in Turaev's paper, and a proof that the surface genus must be greater than 1 . This condition is essential in the discussion of the next natural problem.

Problem. Let $F_{g}$ be a fixed surface of genus $g \geq 2$. How many different (i.e. nonhomeomorphic) closed oriented 3 -manifolds which fiber over $S^{1}$ with $F_{g}$ as fiber become homeomorphic after crossing with $S^{1}$ ?

Our main result concerning the above problem is the following:

TheORem 2. Let $F_{g}$ be a fixed closed oriented surface of genus $g \geq 2$. Then there are at least $\phi(4 g+2)$ nonhomeomorphic 3-manifolds which fiber over $S^{1}$ with $F_{g}$ as fiber and which become homeomorphic after crossing with $S^{1}$.

Here $\phi(-)$ is the Euler function, i.e., $\phi(n)$ is the number of integers $q$, $0 \leq q \leq n$, such that $q$ and $n$ are relatively prime.

Proof of Theorem 2. Let $F_{g}$ be a closed oriented surface of genus $g \geq 2$. By [H1], the maximal cyclic group which can act (nontrivially) on $F_{g}$ has order $4 g+2$. Let $f: F_{g} \rightarrow F_{g}$ be a periodic homeomorphism generating the action of $Z_{4 g+2}$ on $F_{g}$ (i.e., $f^{4 g+2}=$ id).

It is known (cf. [H1], [BC]) that the quotient space $F_{g} / Z_{4 g+2}$ is a sphere $S^{2}$ and the branched covering $p: F_{g} \rightarrow F_{g} / Z_{4 g+2}$ has indices $(2,2 g+1,4 g+2)$ (cf. [BC, p. 582]). To be more specific, the corresponding triangle group $\Gamma$, which determines the action of $Z_{4 g+2}$, has signature $(0 ; 2,2 g+1,4 g+2)$ (cf. [H1]). This gives the corresponding branched data as observed in [H2, p. 393]. Let $m$ be an integer relatively prime to $4 g+2$, i.e., $(m, 4 g+2)=1$. Consider the homeomorphism $f^{m}: F_{g} \rightarrow F_{g}$. Put $f^{m}=h$. Clearly $h$ is periodic of period $4 g+2$.

ClaIm 1. The homeomorphisms $f$ and $h$ determine topologically inequivalent actions of $Z_{4 g+2}$ on $F_{g}$.

Proof of Claim 1. We will show that $f$ and $h$ are not conjugate in Homeo $_{+}\left(F_{g}\right)$. The equivalence of the actions of $Z_{4 g+2}$ on $F_{g}$ can be analyzed in terms of generating vectors (cf. [Br]). In our case, given the presentation $Z_{4 g+2}=\left\langle x \mid x^{4 g+2}=1\right\rangle$, the generating vectors for the action determined by $f$ are given by $\left(x^{2 q+1}, x^{2 q}, x\right)$. 
For the action determined by $h$ the generating vectors are $\left(x^{(2 q+1) m}\right.$, $\left.x^{2 q m}, x^{m}\right)$. Since our equivalence of group actions is simply conjugation (the identity automorphism of $Z_{4 q+2}$ ), the generating vectors are different and the actions are inequivalent. On the other hand, it is well known (cf. $[\mathrm{N}]$, $[\mathrm{S}]$ ) that the actions of cyclic groups on $F_{g}$ are classified by the "fixed point data" (or equivalently, by the branched point data).

Let $\varphi: F_{q} \rightarrow F_{q}$ be a homeomorphism and let $M_{\varphi}$ be the mapping torus of $\varphi$, i.e.,

$$
M_{\varphi}=F_{g} \times[0,1] / \sim,
$$

where $\sim$ is the equivalence relation identifying $F_{g} \times\{0\}$ with $F_{g} \times\{1\}$ via the homeomorphism $\varphi$.

Now, if $\varphi$ is periodic, then $M_{\varphi}$ is a Seifert fibered space with the Seifert invariants uniquely determined by the fixed point data of $\varphi$ (cf. [S, pp. 390-391]). In particular, inequivalent actions, generated by $f$ and $f^{m}$, lead to different manifolds $M_{f}$ and $M_{f^{m}}$. Indeed, the manifolds $M_{f}$ and $M_{f^{m}}$ are distinguished by their Seifert invariants.

Claim 2. $M_{f} \times S^{1} \approx M_{f m} \times S^{1}$ for each $m$ with $(m, 4 g+2)=1$.

Proof of Claim 2. This claim is definitely known. Its proof (in a different notation) is given in [CR, p. 258]. However, for completeness, we outline here the argument from $[\mathrm{CR}]$.

Let $S^{1}$ be the circle equipped with the standard free action of a finite cyclic group. Then the manifolds $M_{f}$ and $M_{h}$ are homeomorphic to the twisted products

$$
M_{f}=S^{1} \underset{\langle f\rangle}{\times} F_{q}, \quad M_{h}=S^{1} \underset{\langle h\rangle}{\times F_{q}},
$$

where $\langle f\rangle$ and $\langle h\rangle$ are the cyclic groups of order $4 q+2$ generated by $f$ and $h$, respectively. Both $M_{f}$ and $M_{h}$ admit natural actions of $Z_{4 g+2}$. Namely, if $q m=1$, then the action of $Z_{4 g+2}$ on $M_{f}$ is generated by $f^{m}$ and the action on $M_{h}$ is generated by $f^{q}$. Both of these actions extend to actions of $S^{1}$. In particular, these actions are generated by homeomorphisms isotopic to the identity. As a consequence, we have

$$
S^{1} \underset{\left\langle f^{m}\right\rangle}{\times} M_{f}=S^{1} \times M_{f}, \quad S^{1} \underset{\left\langle f^{q}\right\rangle}{\times} M_{h}=S^{1} \times M_{h} .
$$

Consider now the action of $Z_{4 g+2} \times Z_{4 g+2}$ on the manifold $S^{1} \times S^{1} \times F_{g}$. This action is generated by $f$ and $f^{m}$. Namely, $\langle f\rangle$ acts on the second and third factor, and $\left\langle f^{m}\right\rangle$ on the first and third factor. Clearly these actions commute. Now factoring out these actions one gets 


$$
\begin{aligned}
& {\left[\left(S^{1} \times S^{1} \times F_{q}\right) /\langle f\rangle\right] /\left\langle f^{q}\right\rangle=S^{1} \underset{\left\langle f^{m}\right\rangle}{\times} M_{f}=S^{1} \times M_{f},} \\
& {\left[\left(S^{1} \times S^{1} \times F_{q}\right) /\left\langle f^{q}\right\rangle\right] /\langle f\rangle=S^{1} \underset{\left\langle f^{q}\right\rangle}{\times} M_{h}=S^{1} \times M_{h},}
\end{aligned}
$$

and hence $S^{1} \times M_{f}=S^{1} \times M_{h}$ as claimed.

REMARK. By using the results in $[\mathrm{Br}]$ it is probably possible to determine all nonhomeomorphic 3-manifolds which fiber over $S^{1}$ with fiber $F_{g}, g=2,3$, and which become homeomorphic after stabilization by $S^{1}$.

Stabilization by even-dimensional spheres. Our main result is the following

THEOREM 3. Let $M^{3}, N^{3}$ be closed, oriented geometric 3-manifolds. Then $M^{3} \times S^{2 k} \approx N^{3} \times S^{2 k}, k \geq 1$, is equivalent to $M^{3} \approx N^{3}$.

Proof. Assume first that $\pi_{1}\left(M^{3}\right)$ is infinite. The existence of a homeomorphism $M^{3} \times S^{2 k} \approx N^{3} \times S^{2 k}$ implies that $\pi_{1}\left(M^{3}\right)=\pi_{1}\left(N^{3}\right)$ and hence $M^{3}$ is homotopy equivalent to $N^{3}$ (i.e., $M^{3} \simeq N^{3}$ ). This is because we can take $M^{3}$ and $N^{3}$ to be irreducible and therefore aspherical. Geometric aspherical 3-manifolds are determined by their fundamental groups (cf. [KS1, p. 738]) and hence $M^{3} \approx N^{3}$ in this case.

Now assume that $\pi_{1}\left(M^{3}\right)$ is finite. Again the isomorphism $\pi_{1}\left(M^{3}\right)=$ $\pi_{1}\left(N^{3}\right)$ implies $M^{3} \approx N^{3}$ unless $M^{3}$ and $N^{3}$ are lens spaces. Suppose then that $M^{3}=L$ is a lens space. We consider the case $k=1$ first. A simple computation of $H_{3}\left(L \times S^{2} ; \mathbb{Z}\right)$ gives

$$
H_{3}\left(L \times S^{2} ; \mathbb{Z}\right) \cong H_{1}(L ; \mathbb{Z}) \otimes H_{2}\left(S^{2}\right) \oplus H_{3}(L ; \mathbb{Z}) \otimes H_{0}\left(S^{2} ; \mathbb{Z}\right) \cong Z_{k} \oplus \mathbb{Z} .
$$

It follows that the map

$$
f: M^{3} \stackrel{i}{\longrightarrow} M^{3} \times S^{2} \stackrel{h}{\underset{\approx}{\longrightarrow}} N^{3} \times S^{2} \stackrel{p}{\longrightarrow} N^{3}
$$

has degree \pm 1 , where $i$ is the natural inclusion and $p$ is the projection. Since $\operatorname{deg}(f)= \pm 1, f$ is a homotopy equivalence (cf. [C, p. 95]).

Let $\tau_{0}=\tau(f) \in \mathrm{Wh}\left(\pi_{1}\left(N^{3}\right)\right)$ be the Whitehead torsion of $f$. We will show that $\tau_{0}=0$, which implies $M^{3} \approx N^{3}$ (cf. [C, p. 100]). Let $j: N^{3} \hookrightarrow$ $N^{3} \times D^{3}$ be the standard embedding $j(x)=(x, 0)$. Consider the homotopy equivalence $\bar{f}=j \circ f: M^{3} \rightarrow N^{3} \rightarrow N^{3} \times D^{3}$. It can be approximated by an embedding $i$ (cf. [W1, Cor. 11.34]) and in fact by a smooth one (cf. [M1, p. 579]). A simple calculation (cf. [M1, p. 579]) shows that the normal bundle of this embedding is trivial. In particular, the closed disk bundle (its total space) is given by $M^{3} \times D^{3}$. The complement of the disk bundle gives an $h$-cobordism $\left(W ; W_{0} ; W_{1}\right)$ between $W_{0}=M^{3} \times S^{2}$ and $W_{1}=N^{3} \times S^{2}$. The Whitehead torsion $\tau\left(W ; W_{0}\right)$ of this $h$-cobordism is equal to $\tau_{0}$. 
Let $\Delta\left(W_{0}\right), \Delta\left(W_{1}\right)$ be the Reidemeister torsions of $W_{0}$ and $W_{1}$, respectively, as defined in [M2, pp. 404-405]. By Theorem 12.8 in [M2] we have

$$
\Delta\left(W_{0}\right) \sim u^{2} \Delta\left(W_{1}\right),
$$

where $u$ is some unit in the group ring $\mathbb{Z}\left[\pi_{1}\left(M^{3}\right)\right]$. Since $W_{1}=N^{3} \times S^{2} \approx$ $W_{0}=M^{3} \times S^{2}$, we have $\Delta\left(W_{0}\right) \sim \Delta\left(W_{1}\right)$, which implies that $u^{2} \sim 1$. But $u^{2} \sim 1$ is equivalent to the triviality of the Whitehead torsion $\tau\left(W ; W_{0}\right)$, again by Theorem $12.8 \mathrm{in}$ [M2]. This means that $\tau_{0}=0$ and hence $\tau(f)=0$ and $M^{3} \approx N^{3}$ as claimed.

Now we consider the case $k \geq 2$, i.e., we have a homeomorphism

$$
M^{3} \times S^{2 k} \approx N^{3} \times S^{2 k},
$$

where $M^{3}, N^{3}$ are lens spaces. As in the case $k=1$, there is a homotopy equivalence $f: M^{3} \rightarrow N^{3}$. Again we approximate $i \circ f: M^{3} \rightarrow N^{3} \times D^{3}$ by a smooth embedding with trivial normal bundle. Let $k: M^{3} \rightarrow N^{3} \times D^{3}$ be such an embedding. Consider the embedding $k^{\prime}: M^{3} \rightarrow N^{3} \times D^{3} \times D^{2 k-2}$ given by $k^{\prime}(x)=(k(x), 0)$. The normal bundle for $k^{\prime}$ is trivial, which leads to an $h$-cobordism $\left(W ; W_{0}, W_{1}\right)$ with $W_{0}=M^{3} \times S^{2 k}, W_{1}=N^{3} \times S^{2 k}$ and $\tau\left(W ; W_{0}\right)=\tau(f)$. The same argument as in the case $k=1$ shows $\tau(f)=0$ and completes the proof of Theorem 3 .

\section{Stabilization by odd-dimensional spheres}

THEOREM 4. Let $M^{3}, N^{3}$ be closed, oriented geometric 3-manifolds. Then $M^{3} \times S^{2 k+1} \approx N^{3} \times S^{2 k+1}, k \geq 1$, is equivalent to:

(a) $M^{3} \approx N^{3}$ if $M^{3}$ is not a lens space.

(b) $M^{3} \simeq N^{3}$ if $M^{3}$ is a lens space and $k>1$, and $\pi_{1}\left(N^{3}\right) \cong \pi_{1}\left(M^{3}\right)$ if $M^{3}$ is a lens space and $k=1$.

Proof. Part (a) follows directly from the isomorphism $\pi_{1}\left(M^{3}\right) \cong \pi_{1}\left(N^{3}\right)$. The second part of $(\mathrm{b})$ is a consequence of result in $[\mathrm{M} \ell]$, more precisely, $[\mathrm{M} \ell$, (41), p. 19]. We are then left with the first part of (b). Given a homeomorphism $M^{3} \times S^{2 k+1} \approx N^{3} \times S^{2 k+1}, k>1$, we obtain a homotopy equivalence $f: M^{3} \rightarrow N^{3}$ between two lens spaces (as in the proof of Theorem 3). Conversely, assume $f: M^{3} \rightarrow N^{3}$ is a homotopy equivalence. Then

$$
f \times \operatorname{id}_{S^{2 k+1}}: M^{3} \times S^{2 k+1} \rightarrow N^{3} \times S^{2 k+1}
$$

is a simple homotopy equivalence because

$$
\tau\left(f \times \operatorname{id}_{S^{2 k+1}}\right)=\chi\left(S^{2 k+1}\right) i_{*} \tau(f)+\chi\left(M^{3}\right) j_{*} \tau\left(\operatorname{id}_{S^{2 k+1}}\right)
$$

(cf. [C, 23.2, p. 77]). 
Now the Sullivan-Wall surgery exact sequence (cf. [W1, p. 107])

$$
\begin{aligned}
\rightarrow\left[\Sigma\left(M^{3} \times S^{2 k+1}\right) ; G / \text { Top }\right] \stackrel{\theta_{2 k+1}}{\longrightarrow} L_{2 k+1}^{s}\left(\pi_{1}\left(M^{3}\right)\right) \stackrel{\vartheta}{\rightarrow} S_{\text {Top }}\left(N^{3} \times S^{2 k+1}\right) \\
\stackrel{\eta}{\longrightarrow}\left[N^{3} \times S^{2 k+1} ; G / \text { Top }\right] \stackrel{\theta_{2 k}}{\longrightarrow} L_{2 k}^{s}\left(\pi_{1}\left(N^{3}\right)\right)
\end{aligned}
$$

easily implies that the class of $f \times \operatorname{id}_{S^{2 k+1}}$ is trivial in the structure set $S_{\text {Top }}\left(N^{3} \times S^{2 k+1}\right)$. This is because:

(i) $f$ being a homotopy equivalence is normally cobordant to the identity (cf. [KS2], and hence $f \times \operatorname{id}_{S^{2 k+1}}$ is normally cobordant to the identity as well (i.e., $\eta\left(f \times \operatorname{id}_{S^{2 k+1}}\right)=0$ ).

(ii) The group $L_{2 k+1}^{s}\left(\pi_{1}\left(N^{3}\right)\right)$ is either 0 or $Z_{2}$ (cf. [W2, p. 33]). In the case $L_{2 k+1}^{s}\left(\pi_{1}\left(N^{3}\right)\right) \cong Z_{2}$, it turns out that $\operatorname{im} \theta_{2 k+1} \cong Z_{2}$. This leads to the triviality of $f \times \operatorname{id}_{S^{2 k+1}}$ in $S_{\text {Top }}\left(N^{3} \times S^{2 k+1}\right)$ as claimed. But the triviality of $f \times \mathrm{id}_{S^{2 k+1}}$ in $S_{\text {Top }}\left(N^{3} \times S^{2 k+1}\right)$ simply means that $f \times \mathrm{id}_{S^{2 k+1}}$ is homotopic to a homeomorphism. In particular, $N^{3} \times S^{2 k+1} \approx M^{3} \times S^{2 k+1}$.

\section{Dropping the geometricity assumption for 3-manifolds with fi-} nite fundamental groups. In this section we look closer at 3-manifolds with finite fundamental groups. To be more specific, we consider 3-manifolds of the form $S^{3} / G$, where $G$ is a finite group acting freely (not necessarily linearly) on $S^{3}$. It turns out, as announced in [R] (cf. [L]), that $G$ has to be a subgroup of $\mathrm{SO}(4)$. Many of these groups have to act linearly (cf. $[\mathrm{R}]$ ), but it is still a conjecture that all of them do. A complete list of corresponding groups $G$ (up to isomorphism with the direct product with a cyclic group of coprime order) is given in Table 1 of [Th]. The manifold $M^{3}=S^{3} / G$ with an arbitrary free action of the group $G$ from the list in [Th] will be called a fake spherical space form.

The following (cf. [JK, Theorem 1]) will be useful in our considerations.

FACT 5. Let $f: M^{3} \rightarrow N^{3}$ be a homotopy equivalence of oriented 3manifolds. Then the normal invariant $\eta(f) \in\left[N^{3} ; G /\right.$ Top $]$ is trivial.

REMARK. It has been proved in [Th] that every fake spherical space form $S^{3} / G$ is homotopy equivalent to a linear one.

Let us consider first the stabilization of fake spherical space forms by odd-dimensional spheres. Let $M^{3}, N^{3}$ be fake spherical space forms. Then clearly $M^{3} \times S^{1} \approx N^{3} \times S^{1} \Leftrightarrow M^{3}$ is $h$-cobordant to $N^{3}$. Therefore, we assume $k>1$ in $S^{2 k+1}$.

Theorem 6. Let $M^{3}, N^{3}$ be closed oriented (prime) 3-manifolds.

(a) If $\left|\pi_{1}\left(M^{3}\right)\right|=\infty$ or $M^{3}, N^{3}$ are fake spherical space forms, then $M^{3} \times S^{3} \approx N^{3} \times S^{3} \Leftrightarrow \pi_{1}\left(M^{3}\right) \cong \pi_{1}\left(N^{3}\right)$.

(b) $M^{3} \times S^{2 k+1} \approx N^{3} \times S^{2 k+1}, k \geq 2 \Leftrightarrow M^{3} \simeq N^{3}$. 
Proof. (a) Let us consider first manifolds with finite fundamental groups. Write $M^{3}=S^{3} / G_{1}, N^{3}=S^{3} / G_{2}$.

CASE 1: $G_{1}$ nonabelian. In this case there is a unique linear space form $X^{3}=S^{3} / G_{1}$ and homotopy equivalences $f_{1}: X^{3} \rightarrow M^{3}$ and $f_{2}: X^{3} \rightarrow N^{3}$. In particular we have a homotopy equivalence $f: M^{3} \rightarrow N^{3}$. Let $F: W \rightarrow$ $N^{3} \times I$ be a normal cobordism between $f$ and the identity $\operatorname{id}_{N^{3}}: N^{3} \rightarrow N^{3}$. Crossing $F$ with id id $_{S^{3}}$ gives a normal map $F \times \mathrm{id}_{S^{3}}$ with a surgery obstruction $\Theta\left(F \times \operatorname{id}_{S^{3}}\right) \in L_{3}^{s}\left(\pi_{1}\left(N^{3}\right)\right)$ (we recall that $\chi\left(S^{3}\right)=0$ so indeed we have an obstruction in the simple surgery group $\left.L^{s}(-)\right)$. It turns out however that this obstruction is trivial by the surgery product formula in [Mo, p. VI]. This means that $f \times \operatorname{id}_{S^{3}}: M^{3} \times S^{3} \rightarrow N^{3} \times S^{3}$ is homotopic to a homeomorphism, in particular $M^{3} \times S^{3} \approx N^{3} \times S^{3}$.

CASE 2: $G_{1}$ abelian. Let $f_{1}: X^{3} \rightarrow M^{3}$ and $f_{2}: Y^{3} \rightarrow N^{3}$ be homotopy equivalences with $X^{3}, Y^{3}$ linear lens spaces. As before, we have $X^{3} \times S^{3} \approx$ $M^{3} \times S^{3}$ and $Y^{3} \times S^{3} \approx N^{3} \times S^{3}$. But $X^{3} \times S^{3} \approx Y^{3} \times S^{3}$ by Theorem 4(b) and hence $M^{3} \times S^{3} \approx N^{3} \times S^{3}$ as well. The converse $M^{3} \times S^{3} \approx N^{3} \times S^{3} \Rightarrow$ $G_{1} \cong G_{2}$ is obvious.

If $\left|\pi_{1}\left(M^{3}\right)\right|=\infty$, then the isomorphism $\pi_{1}\left(M^{3}\right) \cong \pi_{1}\left(N^{3}\right)$ is equivalent to existence of a homotopy equivalence $f: M^{3} \rightarrow N^{3}$. Since $f$ is normally cobordant to the identity, $f \times \operatorname{id}_{S^{3}}$ is homotopic to a homeomorphism by the surgery product formula, as in the finite fundamental group case.

(b) We simply observe that the homeomorphism $M^{3} \times S^{2 k+1} \approx N^{3} \times$ $S^{2 k+1}, k \geq 2$, gives a degree one map $f: M^{3} \rightarrow N^{3}$ inducing an isomorphism $f_{\#}: \pi_{1}\left(M^{3}\right) \rightarrow \pi_{1}\left(N^{3}\right)$. By Lemma 1.1 in [S] this gives a homotopy equivalence $M^{3} \simeq N^{3}$. As before, a homotopy equialence $M^{3} \simeq N^{3}$ leads to the existence of a homeomorphism $M^{3} \times S^{2 k+1} \approx N^{3} \times S^{2 k+1}$ via the surgery product formula from $[\mathrm{Mo}]$.

REMARK 7. By using Fact 5 one can show that Theorem 6(b) holds for all closed (not necessarily prime) oriented 3-manifolds. In Theorem 6(a) for many groups, for example: quaternionic 2-groups $D_{2 q}^{*}$, tetrahedral groups $T_{v}^{*}, v \geq 2$, cyclic groups $Z_{k}$, one can replace $S^{3}$ by a homotopy 3 -sphere $\Sigma^{3}$ in the definition of $M^{3}$ and $N^{3}$, i.e., $M^{3}=\Sigma^{3} / G_{1}, N^{3}=\Sigma^{3} / G_{2}$.

We now consider the following families of 4-periodic finite groups:

1. Generalized quaternionic groups $Q(8 p, q, r)$,

2. Cyclic groups $Z_{k}$,

3. Platonic groups $T_{1}^{*}, O^{*}$ and $I^{*}$.

The particular interest in these groups is because:

1. The groups $Q(8 p, q, r)$ are not subgroups of $\mathrm{SO}(4)$ but potentially can act freely on some homotopy $S^{3}$. 
2. The groups $Z_{k}$ for some $k>3$ can potentially act nonlinearly on $S^{3}$ (cf. $[\mathrm{R}]$ ).

3. There are homotopically exotic actions of $T_{1}^{*}, O^{*}$ and $I^{*}$ (cf. [Th, Corollary on p. 293]).

THEOREM 8. Let $M^{3}=\Sigma^{3} / G_{1}, N^{3}=\Sigma^{3} / G_{2}$ be fake spherical space forms, where $\Sigma^{3}$ is a homotopy 3-sphere and $G_{1}, G_{2}$ are groups from the families $1-3$ above. Then $M^{3} \times S^{2 k} \approx N^{3} \times S^{2 k}, k \geq 1 \Leftrightarrow M^{3}$ and $N^{3}$ are simply homotopy equivalent.

Proof. Assume $M^{3} \times S^{2 k} \approx N^{3} \times S^{2 k}$. Then there is a homotopy equivalence $f: M^{3} \rightarrow N^{3}$. We will show that the Whitehead torsion $\tau(f)$ is trivial. As in the proof of Theorem 3, we obtain an $h$-cobordism $W$ between $M^{3} \times S^{2 k}$ and $N^{3} \times S^{2 k}$ whose torsion is that of $f$. To conclude that $W$ is in fact an $s$-cobordism we argue as in the proof of Theorem 3 . The only new ingredients needed here are:

(a) The involution on the Whitehead group $W h\left(\pi_{1}\left(N^{3}\right)\right)$ is trivial (cf. [KS1, Theorem 2.1]).

(b) The Whitehead group $\mathrm{Wh}\left(\pi_{1}\left(N^{3}\right)\right)$ is torsion free for the groups in question (cf. [KS1]).

On the other hand, if $f: M^{3} \rightarrow N^{3}$ is a simple homotopy equivalence, then

$$
f \times \operatorname{id}_{S^{2 k}}: M^{3} \times S^{2 k} \rightarrow N^{3} \times S^{2 k}, \quad k \geq 1,
$$

is homotopic to a homeomorphism by the surgery product formula in [Mo].

We end this paper with an example which shows that Theorem 3 cannot be extended to manifolds of dimension higher than 3 and that the assertion of Theorem 8 is the best one can hope for.

EXAMPLE 9. There are simple homotopy equivalent but nonhomeomorphic fake lens spaces $L_{1}$ and $L_{2}$ (in every dimension $2 n+1, n \geq 2$ ) such that $L_{1} \times S^{2 k} \approx L_{2} \times S^{2 k}$ for each $k \geq 1$.

Proof. Let $Z_{r}, r \geq 2$, be the finite cyclic group. Let $L_{0}^{s}\left(Z_{r}\right)$ be the surgery obstruction group and let $\widetilde{L}_{0}^{s}\left(Z_{r}\right)$ be the reduced group $\widetilde{L}_{0}^{s}\left(Z_{r}\right)=$ $L_{0}^{s}\left(Z_{r}\right) / L_{0}^{s}(0)$. The group $\widetilde{L}_{0}^{s}\left(Z_{r}\right)$ is torsion free and is detected by the multisignature (cf. [W2]). Let $L_{2}$ be a seven-dimensional linear lens space with $\pi_{1}\left(L_{2}\right) \cong Z_{r}$.

Let $0 \neq \alpha \in \widetilde{L}_{0}^{s}\left(Z_{r}\right)$ be a nontrivial element. Realize $\alpha$ by a normal map

$$
F:\left(W ; \partial_{0} W, \partial_{1} W\right) \rightarrow\left(L_{2} \times I, L_{2} \times\{0\}, L_{2} \times\{1\}\right)
$$

with $\Theta(F)=\alpha \in \widetilde{L}_{0}^{s}\left(Z_{k}\right)$ and $f=\left.F\right|_{\partial_{1} W}: \partial_{1} W \rightarrow L_{1} \times\{1\}$ a simple homotopy equivalence. Put $\partial_{1} W=L_{1}$. Clearly, $L_{1}$ is a fake lens space.

Claim. $L_{1} \not \approx L_{1}$. 
Suppose $L_{1} \approx L_{2}$. Then their $\varrho$-invariants are the same (cf. [W1]). But this would imply $\alpha=0$, a contradiction.

Now applying the surgery product formula, we see that $f \times \operatorname{id}_{S^{2 k}}$ is homotopic to a homeomorphism and hence $L_{1} \times S^{2 k} \approx L_{2} \times S^{2 k}$. By replacing $\widetilde{L}_{0}^{s}\left(Z_{r}\right)$ by $\widetilde{L}_{2}^{s}\left(Z_{r}\right)$ and using the fact that the torsion free part of $\widetilde{L}_{2}^{s}\left(Z_{r}\right)$ is determined by the multisignature (cf. [W2]) we infer that the analogous construction for five-dimensional lens spaces leads to the desired result in all odd dimensions.

Appendix: Proof of Theorem 1. There are two cases to be considered:

CASE 1: $\pi_{1}\left(M^{3}\right)$ is finite.

CASE 2: $\pi_{1}\left(M^{3}\right)$ is infinite.

Moreover, for clarity of our argument, Case 2 is split further into:

CASE 2.A: $M^{3}$ is a "large" Seifert manifold.

CASE 2.B: $M^{3}$ is a "small" Seifert manifold.

Proof of Case 1. The homeomorphism $M^{3} \times S^{1} \approx N^{3} \times S^{1}$ implies that $\pi_{1}\left(N^{3}\right)$ is finite as well. If $\pi_{1}\left(M^{3}\right)$ is nonabelian, then so is $\pi_{1}\left(N^{3}\right)$ and both groups are isomorphic. Now, 3-manifolds with finite nonabelian fundamental groups are classified by those groups (cf. [KS1, p. 737], [TS, p. 567]). If $\pi_{1}\left(M^{3}\right)$ is abelian, then $\pi_{1}\left(N^{3}\right)$ is also abelian and we have two lens spaces (cf. [O]). The homeomorphism $h: M^{3} \times S^{1} \rightarrow N^{3} \times S^{1}$ lifts to a homeomorphism $\widetilde{h}: M^{3} \times \mathbb{R} \rightarrow N^{3} \times \mathbb{R}$. Indeed, if $h_{\#}: Z_{k} \times \mathbb{Z} \rightarrow Z_{k} \times \mathbb{Z}$ is the induced homomorphism on the fundamental groups, then $h_{\#}\left(Z_{k}\right)=Z_{k}$ and $h_{\#}(\mathbb{Z})=\mathbb{Z}$. The existence of a homeomorphism $\widetilde{h}$ implies the existence of an $h$-cobordism between $M^{3}$ and $N^{3}$. Now the Atiyah-Bott fixed point theorem (cf. [M2, Corollary 12.12]) implies that $M^{3}$ and $N^{3}$ are homeomorphic.

Proof of Case 2 (general comments). To simplify the notation, let $G=$ $\pi_{1}\left(M^{3}\right), H=\pi_{1}\left(N^{3}\right)$ and let $h_{\#}: G \times \mathbb{Z} \rightarrow H \times \mathbb{Z}$ be the induced isomorphism. Let $Z(G)$ be the center of $G$.

Claim. If $Z(G)=\{1\}$, then $Z(H)=\{1\}$.

Proof of claim. The center of $G \times \mathbb{Z}$ consists of a copy of $\mathbb{Z}$ (i.e., $\{1\} \times \mathbb{Z}$ in $G \times \mathbb{Z})$. Consider the image $h_{\#}(\{1\} \times \mathbb{Z})$ of the center. If $h_{\#}(\{1\} \times \mathbb{Z})=\{1\} \times \mathbb{Z}$ in $H \times \mathbb{Z}$, then we are done. Suppose then that $h_{\#}(\{1\} \times t)=(p, s), p \in H$, where $t$ is a generator of $\mathbb{Z}$ and $p \neq\{1\}$. The manifold $N^{3}$, being prime, is either a $K\left(\pi_{1}\left(N^{3}\right), 1\right)$-manifold or $S^{1} \times S^{2}$ (cf. [H]). In both cases the fundamental group of $N^{3}$, i.e., $H$, is torsion free (cf. [H]). Let $C$ be the cyclic subgroup of $H$ generated by $p$. It follows that the center of $H \times \mathbb{Z}$ 
contains $\mathbb{Z} \times \mathbb{Z}$. The inverse isomorphism $h_{\#}^{-1}$ sends the center of $H \times \mathbb{Z}$ to the center of $G \times \mathbb{Z}$. This implies that the center of $G$ is nontrivial, which is a contradiction. As a consequence, $h_{\#}(\{1\} \times \mathbb{Z})=\{1\} \times \mathbb{Z} \subset H \times \mathbb{Z}$, which proves the claim.

Now given the claim, in the absence of the center of $G$, the homeomorphism $h: M^{3} \times S^{1} \rightarrow N^{3} \times S^{1}$ lifts to a homeomorphism $\widetilde{h}: M^{3} \times \mathbb{R} \rightarrow$ $N^{3} \times \mathbb{R}$. In particular, we have an isomorphism $h_{\#}: \pi_{1}\left(M^{3}\right) \rightarrow \pi_{1}\left(N^{3}\right)$. This in turn implies the existence of a homeomorphism between $M^{3}$ and $N^{3}$ (cf. [KS1]). Consider now the case of nontrivial center of $\pi_{1}\left(M^{3}\right)$. Without loss of generality, we can assume that $M^{3}$ is irreducible (the only reducible case of $S^{1} \times S^{2}$ is fully understood). Now $M^{3}$ being closed, oriented, irreducible with infinite $\pi_{1}\left(M^{3}\right)$ and nontrivial center is Seifert fibered by the algebraic characterization of Seifert manifolds (cf. $[\mathrm{CJ}],[\mathrm{G}]$ ).

Proof of Case 2.A. We consider the case when $M$ and $N$ are "large" Seifert manifolds (cf. [O, pp. 91-92]). Let $\pi=\pi_{1}(M)$ and $\pi^{\prime}=\pi_{1}(N)$. These groups are given by central extensions

$$
0 \rightarrow C \stackrel{i}{\rightarrow} \pi \stackrel{p}{\rightarrow} \Gamma \rightarrow 0, \quad 0 \rightarrow C^{\prime} \stackrel{i^{\prime}}{\rightarrow} \pi^{\prime} \stackrel{p^{\prime}}{\rightarrow} \Gamma^{\prime} \rightarrow 0
$$

where $C \approx C^{\prime} \approx \mathbb{Z}$. The homomorphisms $i, i^{\prime}$ are the natural inclusions and $p, p^{\prime}$ the projections. Now the Hopf formula (cf. [B, p. 41]) applied to these extensions leads to the following exact sequences in homology of groups (cf. [B, p. 47]):

$$
\begin{aligned}
H_{2}(\pi) & \rightarrow H_{2}(\Gamma) \stackrel{\beta_{*}}{\rightarrow} H_{1}(C) \stackrel{i_{*}}{\rightarrow} H_{1}(\pi) \stackrel{p_{*}}{\rightarrow} H_{1}(\Gamma) \rightarrow 0, \\
H_{2}\left(\pi^{\prime}\right) & \rightarrow H_{2}\left(\Gamma^{\prime}\right) \stackrel{\beta_{*}^{\prime}}{\rightarrow} H_{1}(C) \stackrel{i_{*}^{\prime}}{\rightarrow} H_{1}\left(\pi^{\prime}\right) \stackrel{p_{*}^{\prime}}{\rightarrow} H_{1}\left(\Gamma^{\prime}\right) \rightarrow 0 .
\end{aligned}
$$

Suppose that, say, $M$ does not fiber over $S^{1}$ with periodic monodromy. This is equivalent to the condition that the class $\langle h\rangle$ represented by the generator $h$ of $C$ is of finite order in $H_{1}(\pi)$ (cf. [O, p. 122] and [J, VI.31, p. 106]). This is then equivalent to the condition $\operatorname{im} \beta_{*} \neq 0$ in the exact homology sequence.

Let $f: M \times S^{1} \rightarrow N \times S^{1}$ be a homeomorphism. The natural extensions

$$
\begin{gathered}
0 \rightarrow C \times \mathbb{Z} \stackrel{i \times \mathrm{id}}{\longrightarrow} \pi \times \mathbb{Z} \stackrel{\bar{p}}{\rightarrow} \Gamma \rightarrow 0, \\
0 \rightarrow C^{\prime} \times \mathbb{Z} \stackrel{i^{\prime} \times \mathrm{id}}{\longrightarrow} \pi^{\prime} \times \mathbb{Z} \stackrel{\overline{p^{\prime}}}{\rightarrow} \Gamma^{\prime} \rightarrow 0
\end{gathered}
$$

lead to the commutative diagram

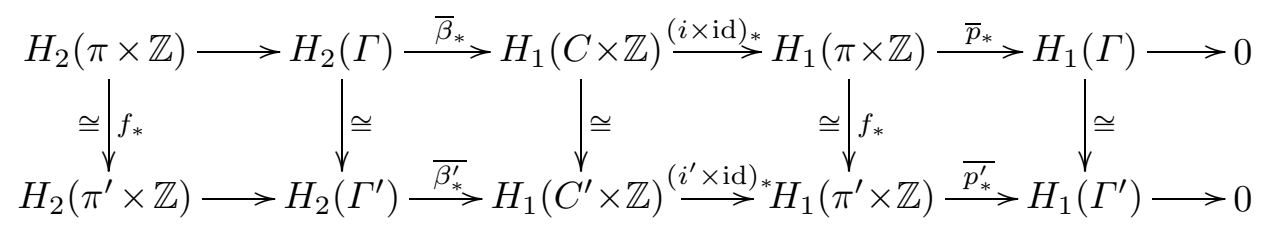


Since $\operatorname{im} \bar{\beta}_{*}$ can be naturally identified with $\operatorname{im} \beta_{*}$ (and the same for $\overline{\beta_{*}^{\prime}}$ ), it follows that $\operatorname{im} \beta_{*} \neq 0$ implies $\operatorname{im} \beta_{*}^{\prime} \neq 0$. This shows that the isomorphism $f$ on $C \times \mathbb{Z} \rightarrow C^{\prime} \times \mathbb{Z}$ must be a product isomorphism. As a consequence, the homeomorphism $f: M \times S^{1} \rightarrow N \times S^{1}$ can be lifted to a homeomorphism $\widetilde{f}: M \times \mathbb{R} \rightarrow N \times \mathbb{R}$. This, however, means that $\pi \approx \pi^{\prime}$ and hence $M \approx N$.

Proof of Case 2.B. We now assume that $M$ (or $N$ ) is a "small" Seifert manifold (cf. [O, pp. 91-92]). In the notation of [O] the possibilities for these manifolds are classes (iii), (iv) or (ix) in [O, pp. 124-125] (we recall that our manifolds are orientable). Class (iii) consists of a single manifold $X$ which is a torus bundle over $S^{1}$ with periodic monodromy of period 2. Class (ix) consists of manifolds which either have a fundamental group with trivial center or are homeomorphic to $X$. Since the case of trivial center has already been considered, we are left with the manifold $X$ and class (iv). For the manifolds in class (iv), the class $\langle h\rangle$ has finite order in $H_{1}(\pi)$ (cf. [O, p. 124]). In view of [O, Theorem 12.10, p. 131], the argument used for the case of "large" Seifert manifolds shows that if $M, N$ are in class (iv) and $M \times S^{1} \approx N \times S^{1}$, then $M \approx N$. For the manifold $X$ in (iii), the corresponding class $\langle h\rangle$ is of infinite order in $H_{1}(\pi)$ and hence $X \not \approx M$ for any $M$ in class (iv). As a consequence, if $M$ is a "small" Siefert manifold and $M \times S^{1} \approx N \times S^{1}$, then $M \approx N$.

Finally, if $M$ is a $T^{2}$ bundle over $S^{1}$ with periodic monodromy and $N$ is a surface bundle over $S^{1}$ with periodic monodromy with $M \times S^{1} \approx N \times S^{1}$, then it follows that $N$ is a $T^{2}$ bundle over $S^{1}$ as well. Given the classification of $T^{2}$ bundles over $S^{1}$ with periodic monodromy in [H, Ex. 12.3, p. 122], one concludes that if $M, N$ are such bundles with $M \times S^{1} \approx N \times S^{1}$, then $M \approx N$. Indeed, if $M \not \nsim N$, then $H_{1}\left(M \times S^{1} ; \mathbb{Z}\right) \not H_{1}\left(N \times S^{1} ; \mathbb{Z}\right)$.

\section{References}

[Br] S. A. Broughton, Classifying finite group actions on surfaces of low genus, J. Pure Appl. Algebra 69 (1990), 233-270.

[B] K. S. Brown, Cohomology of Groups, Grad. Texts in Math. 87, Springer, 1982.

[BC] F. Bujolance and M. Conder, On cyclic groups of automorphisms of Riemann surfaces, J. London Math. Soc. (2) 59 (1999), 573-584.

[CJ] A. Casson and D. Jungries, Convergence groups and Seifert fibered 3-manifolds, Invent. Math. 118 (1994), 441-456.

[C] M. M. Cohen, A Course in Simple Homotopy Theory, Springer, New York, 1973.

[CR] P. Conner and F. Raymond, Derived actions, in: Proc. 2nd Conf. on Compact Transformation Groups (Amherst, MA, 1971), Lecture Notes in Math. 299, Springer, Berlin, 1972, 237-310.

[G] D. Gabai, Convergence groups are Fuchsian groups, Ann. of Math. 136 (1992), 447-510.

[H1] W. J. Harvey, Cyclic groups of automorphisms of a compact Riemann surface, Quart. J. Math. Oxford Ser. (2) 17 (1966), 86-97. 
[H2] W. J. Harvey, On branch loci in Teichmüller space, Trans. Amer. Math. Soc. 153 (1971), 387-399.

[H] J. Hempel, 3-manifolds, Ann. of Math. Stud. 86, Princeton Univ. Press, 1976.

[J] W. Jaco, Lectures in Three-Manifold Topology, CBMS Reg. Conf. Ser. in Math. 43, Amer. Math. Soc., Providence, RI, 1980.

[JK] B. Jahren and S. Kwasik, 3-dimensional surgery theory, UNil-groups and the Borel conjecture, Topology 42 (2003), 1353-1369.

[JN] M. Jenkins and W. Neumann, Seifert Manifolds, notes, Univ. of Maryland, 1981.

[KS1] S. Kwasik and R. Schultz, Vanishing of Whitehead torsion in dimension four, Topology 31 (1992), 735-756.

[KS2] - - , All $\mathbb{Z}_{q}$ lens spaces have diffeomorphic squares, ibid. 41 (2002), 321-340.

[L] R. Lee, Semicharacteristic classes, ibid. 12 (1973), 183-199.

[M $\ell \quad$ W. Metzler, Diffeomorphismen zwischen Produkten mit dreidimensional Linsenräumen als Faktoren, Dissertationes Math. 65 (1969).

[M1] J. Milnor, Two complexes which are homeomorphic but combinatorially distinct, Ann. of Math. 74 (1961), 575-590.

[M2] —, Whitehead torsion, Bull. Amer. Math. Soc. 72 (1963), 358-426.

[Mo] J. Morgan, A product formula for surgery obstruction, Mem. Amer. Math. Soc. 201 (1978).

[N] J. Nielsen, Die Struktur periodischer Transformationen von Flächen, Denske Vid. Selsk. Mat.-Fys. Medd. 15 (1937), no. 1, 77 pp.

[O] P. Orlik, Seifert Manifolds, Lecture Notes in Math. 291, Springer, 1972.

[R] H. Rubinstein, An algorithm to recognize the 3-sphere, in: Proc. Internat. Congress Math. (Zürich, 1994), Birkhäuser, 1995, 601-611.

[S] G. A. Swarup, On a theorem of C. B. Thomas, J. London Math. Soc. (2) 8 (1974), $13-21$.

[Sy] P. Symonds, The cohomology representation of an action of $C_{p}$ on a surface, Trans. Amer. Math. Soc. 306 (1988), 389-400.

[Th] C. B. Thomas, Homotopy classification of free actions by finite groups on $S^{3}$, Proc. London Math. Soc. 49 (1980), 284-297.

[TS] W. Threlfall und H. Seifert, Topologische Untersuchung der Diskontinuitätsbereiche endlicher Bewegungsgruppen des dreidimensional sphärischen Raumes (Schluß), Math. Ann. 107 (1932), 543-586.

[T] W. Thurston, Three dimensional manifolds, Kleinian groups and hyperbolic geometry, Bull. Amer. Math. Soc. 6 (1982), 357-382.

[Tu] V. Turaev, Towards the topological classification of geometric 3-manifolds, in: Topology and Geometry-Rohlin Seminar, Lecture Notes in Math. 1346, Springer, 1988, 291-323.

[W1] C. T. C. Wall, Surgery on Compact Manifolds, Academic Press, 1970.

[W2] - On classification of hermitian forms: VI, Group rings, Ann. of Math. 103 (1976), 1-80.

Department of Mathematics

Tulane University

New Orleans, LA 70118, U.S.A.

E-mail: kwasik@math.tulane.edu
Department of Mathematics

Gdańsk University Wita Stwosza 57

80-952 Gdańsk, Poland E-mail: wrosicki@math.univ.gda.pl

Received 2 December 2003;

in revised form 28 June 2004 